\title{
MUERTE FETAL ANTEPARTO
}

\section{Doctor Miguel Hurtado}

\section{Introducción}

Hemos escogido para el presente trabajo un tema, que tal vez por su complejidad, debía despertar gran interés en todos los que en una u otra forma están vinculados con la obstetricia. Sin embargo, como más adelante observamos, tal interés se ha trocado si se quiere en despreocupación en el manejo de la paciente que nos consulta con feto muerto.

Una demostración de ello ha sido la falta en las historias de una buena anamnesis, la que hubiera facilitado una mejor orientación hacia el diagnóstico etiológico, precisión del tiempo de muerte fetal, etc., que complementada con un estudio cuidadoso, hubiera permitido seguir la conducta adecuada. Todo esto, agregado a la baja asistencia a la consulta prenatal, nos hace pensar que podremos conducir mejor este problema cuando obviemos estos inconvenientes.

En la presente revisión nos hemos propuesto desarrollar con preferencia tres aspectos relativos al problema: $1^{\text {9 }}$ La causa de la muerte fetal; $2^{\circ}$ Su diagnóstico, y $3^{\circ}$ La conducta que ha de seguirse.

\section{Material y métodos}

El material del presente trabajo lo constituyen las historias de fetos nacidos muertos en el Instituto Materno Infantil "Concepción Villaveces de Acosta" (5) entre el 19 de marzo de 1959 y el 28 de febrero de 1963. Queremos aclarar que de tales historias escogimos todas aquellas de fetos muertos de más de 1.500 grs, y cuyo fallecimiento ocurrió antes de que se iniciara el trabajo de parto.

\section{Incidencia}

Entre los 50.420 partos atendidos en el lapso revisado, nacieron 1.637 mortinatos $(3.24 \%)$, de los cuales escogi$\operatorname{mos} 678 \operatorname{casos}(1.34 \%)$.

\section{Edad de las pacientes}

Agrupamos en el siguiente cuadro la frecuencia de muerte fetal anteparto en relación a la edad de la madre, apreciándose entre nosotros una mayor frecuencia entre los 21 y los 25 años, en contraste con el promedio de 30.1 años que dan los norteamericanos (10). 


\begin{tabular}{|c|c|c|c|}
\hline & & Casos & $\%$ \\
\hline Menos de 15 años & $\ldots$ & 2 & 0.29 \\
\hline De 15 a 20 años & $\ldots$ & 153 & 22.63 \\
\hline De 21 a 25 años. & $\ldots$ & 172 & 25.44 \\
\hline De 26 a 30 años. & $\ldots$ & 137 & 20.26 \\
\hline De 31 a 35 años. & $\ldots$ & 111 & 16.42 \\
\hline De 36 a 40 años. & $\ldots$ & 78 & 11.53 \\
\hline Más de 40 años. & $\ldots$ & 22 & 3.25 \\
\hline Sin dato ...... & $\ldots$ & 1 & 0.14 \\
\hline
\end{tabular}

La paciente de menor edad fue de 14 años y la mayor edad fue de 47 años.

\section{Paridad}

Según el número de gestaciones, las pacientes se agruparon así:

\begin{tabular}{|c|c|c|}
\hline & Casos & $\%$ \\
\hline Primíparas .. & 209 & 30.91 \\
\hline Secundíparas . . . . . & 104 & 15.38 \\
\hline Multíparas ...... & 362 & 53.55 \\
\hline Sin dato .......... & 1 & 0.14 \\
\hline
\end{tabular}

Como se aprecia en el cuadro anterior, la mayor incidencia de muerte fetal sucede en las multíparas, seguidas de las primíparas.

Encontramos antecedentes de fetos muertos sólo en 5 multíparas:

\begin{tabular}{lllll}
\hline 1 & Feto muerto $\ldots \ldots$ & 1 & caso \\
2 & Fetos muertos $\ldots$ & 3 & casos \\
3 & Fetos muertos $\ldots$ & $\ldots$ & 1 & caso \\
\hline
\end{tabular}

O sea una sola observación de muerte habitual, si se entiende por tal, la que sucede 3 o más veces en una misma paciente.
Gestación en semanas

Calculamos la edad del embarazo ateniéndonos al dato de la última regla dado por las pacientes, y las agrupamos así :

\begin{tabular}{lrr}
\hline & Casos & \multicolumn{1}{c}{$\%$} \\
\hline De 28 a 30 semanas & 52 & 7.69 \\
De 31 a 35 semanas & 118 & 17.45 \\
De 36 a 40 semanas & 455 & 67.30 \\
Más de 40 semanas & 50 & 7.39 \\
Sin dato & 1 & 0.14 \\
\hline
\end{tabular}

Como bien se puede apreciar, es más frecuente la muerte fetal anteparto después de las 36 semanas.

Tipo de embarazo

\begin{tabular}{lrrr}
\hline & & Casos & \multicolumn{1}{c}{$\%$} \\
\hline Embarazo único & $\ldots$ & 664 & 98.22 \\
Embarazo gemelar & $\ldots$ & 12 & 1.77 \\
\hline
\end{tabular}

De los 12 casos de embarazo gemelar, en 8 sólo murió uno de los gemelos; en los 4 restantes murieron ambos, pero únicamente 2 de ellos se incluyen en el trabajo por tener peso por encima de los 1.500 grs.

Diagnóstico clínico de muerte fetal

Es este uno de los apartes de mayor importancia en la presente revisión. Hemos tenido siempre como elementos diagnósticos la auscultación fetal y la investigación del signo de Negri, éste último un signo tardío pues es sinónimo de maceración fetal. En pocas ocasiones se insistió en los datos dados por la paciente y se despreció un signo constante y precoz como es la calostrorrea, al igual 
que el radiodiagnóstico, exploración esta de gran utilidad por dar signos precoces (9).

Se hizo el diagnóstico en 664 casos $(98.22 \%)$ así:

\begin{tabular}{lcc}
\hline & Casos & $\%$ \\
\hline $\begin{array}{l}\text { Por sólo ruidos fetales } \\
\text { Por ruidos fetales y } \\
\text { Negri . . . . . . . }\end{array}$ & 208 & 68.67 \\
\hline
\end{tabular}

El diagnóstico estuvo complementado así :

Con dato de la paciente $290 \quad 43.67$

Con rayos $X \ldots \ldots \quad 17 \quad 2.56$

Con calostrorrea ... $4 \quad 4 \quad 0.60$

De las radiografías, 5 fueron negativas para muerte fetal; únicamente en 3 se anotó el signo de Spalding.

Haremos acá una descripción de los elementos diagnósticos con su valor práctico; son ellos signos subjetivos, objetivos y exploratorios $(4,12,13,16)$.

\section{Signos subjetivos}

Hacen parte las más de las veces del motivo de consulta de las pacientes, y se refieren a la desaparición de la sintomatología general vagal; a la pérdida de la sensación de movimientos fetales; a la disminución del volumen uterino y a la calostrorrea $(4,12,13,16)$. Estos 2 últimos signos por ser igualmente exploratorios los tendremos en cuenta más adelante.

\section{Signos objetivos y exploratorios}

Pudiéramos considerarlos clínicos y paraclínicos o de laboratorio; hacemos un resumen de ellos:
Asucultación fetal. Debe ser francamente negativa. Es el método más usado, no sólo por hacer parte del examen obstétrico rutinario sino porque además, siendo el de más fácil realización, sus errores sólo están ligados con las alteraciones del tono uterino, sea por sobredistención o por hipertonía. Los ruidos de vecindad son siempre fáciles de diferenciar, y se recuerda la posibilidad, en sentido opuesto, de confundir una taquicardia materna con el corazón fetal y creer que el feto esté vivo. Aquí la diferencia la da la embriocardia.

Signo de Negri. Signo clínico de gran valor diagnóstico; está dado por la crepitación de los huesos de la bóveda craneana fetal cuando hay licuefacción de la masa encefálica. Es un signo constante pero tardío $(4,12,13,16)$.

Calostrorrea. Signo constante y pre$\mathrm{coz}$ que en muy pocas ocasiones se explora, está dado por la secreción de calostro, debido a la deplesión hormonal cuando se interrumpe la actividad placentaria $(4,12,13,16)$. Puede ser también un signo subjetivo.

Disminución del volumen uterino. Es un signo subjetivo tardío que está dado por la disminución del contenido uterino en sus procesos de reabsorción tanto fetal como de líquido amniótico $(4,12,13,16)$. Su exploración ha de hacerse comparativamente, en los días siguientes, teniendo en cuenta que en estos casos, el útero disminuye hasta 2 centímetros por día; retrospectivamente, por la desproporción por defecto entre la altura uterina y la amenorrea.

Pérdida de la actitud fetal. Ocasionalmente la palpación nos alerta para el 
diagnóstico de muerte fetal cuando encontramos fácilmente la pérdida de la actitud fetal, la cual se acentúa con los cambios posturales de la madre; se requiere la mavoría de las veces que la paciente sea delgada y que la cantidad de líquido amniótico sea normal.

Amniocentesis. (Método de E. M. Baldi) (16). Es un método preconizado por los norteamericanos con fines diagnósticos (10), basados en los cambios de la apariencia del líquido amniótico. Incluso la presencia de sangre en el líquido (Reacción de Adler) (16), ha sido descrita como signo de muerte fetal. Esta reacción está dada por una coloración verde intensa agregando al líquido de la punción unas gotas de ácido acético, bencidina acética y agua oxigenada. Entre nosotros no hubo experiencia al respecto.

Reacciones biológicas. No las consideramos, no sólo por no haberse practicado entre nosotros sino porque su valor ha sido discutido en el tema que tratamos. Mas, para algunos (4, 12, $13,16)$, pueden prestar utilidad al ser negativas cualitativamente $\mathrm{y}$ ratificarse su eficacia para el diagnóstico de la muerte fetal, con una técnica cuantitativa.

\section{Radiodiagnóstico}

Es tal vez este método exploratorio en el cual se han descrito mayor número de signos de muerte fetal, lo cual justifica su uso. De ellos los más conocidos los enumeraremos a continuación:

Signo de Spalding. Está dado por el acabalgamiento de los huesos del crá- neo, debido a la par que el de Negri a la licuefacción de la masa cerebral. Es un signo tardío pero constante. Tiene valor, lo mismo que otros signos, con integridad de las membranas y en ausercia de contracciones de parto (4, $12,13,16$ ).

Signo de Horner. Consiste en la asimetría acentuada del cráneo. Es igualmente un signo tardío (13).

Signo de Spangler. Consiste en el achatamiento del cráneo fetal (13).

Signo de Iungmann. Está dado por el enderezamiento de la columna dorsolumbar del feto muerto, por atonía de la masa muscular dorsolumbar (13).

Gas intrafetal in útero. Está dado por la presencia de gas intraperitoneal, extravascular e intravascular (9). Fue descrito inicialmente por Roberts en 1944. Es considerado por los norteamericanos como el único signo radiológico patognomónico de muerte fetal in útero y de gran valor por su precocidad (9). Samuel y Gunn estiman que nunca el gas intravascular se encuentra en los vasos pulmonares y cerebrales (7).

Signo del halo. Fue descrito en 1947 por Deuel y está constituído por la presencia de una aureola pericraneana que corresponde a una acumulación de aire en el espacio que separa el cuero cabelludo de los tejidos grasos suyacentes $(2,7)$.

Amniografía. Se interpreta como signo de muerte fetal cuando no se demuestra la deglución por parte del feto del medio de contraste utilizado (13). 


\section{Electrocardiografía y}

electroencefalografía fetal

Este procedimiento es tal vez el más usado entre los norteamericanos (11, 18), quiencs a más del valor que le dan para el diagnóstico de sufrimiento fetal, ya propuesto entre nosotros desde 1957 (14), lo han encontrado de gran utilidad para el diagnóstico de muerte fetal dada su fácil ejecución y por el margen de seguridad diagnóstica (7).

\section{Investigación de la causa de} muerte fetal por laboratorio

Con este objeto, sólo se utilizó el laboratorio en 198 casos (29.28\%), índice que corrobora el desinterés ya anotado al comienzo del trabajo. Algo más; en ellos está incluído el único caso de anatomía patológica con fines diagnósticos, cual fue un estudio de placenta y cabe anotar que dicho estudio careció de utilidad alguna.

\section{Duración del trabajo}

Este dato se obtuvo basados en la anotación que traen las historias de fecha y hora de iniciación del trabajo, hasta el momento de la expulsión o extracción del feto. Las agrupamos así:

\begin{tabular}{|c|c|c|}
\hline & Casos & $\%$ \\
\hline Menos de 5 horas ... & 145 & 21.44 \\
\hline De 5 a 10 horas... & 277 & 40.97 \\
\hline De 10 a 15 horas ... & 136 & 20.11 \\
\hline De 15 a 20 horas ... & 47 & 6.95 \\
\hline Más de 20 horas ... & 17 & 2.51 \\
\hline Sin trabajo de parto .. & 17 & 2.51 \\
\hline Sin dato ....... & 37 & 5.47 \\
\hline
\end{tabular}

Es decir, la mayor frecuencia fue de 5 a 10 horas.

El menor trabajo fue de 45 minutos y el mayor de 31.50 horas.

Los casos llamados "sin trabajo", aluden a las cesáreas practicadas en pacientes que no habían iniciado trabajo y en las que la entidad causal de la muerte fetal implicaba un riesgo materno.

El trabajo de 31.50 horas lo consideramos errado, ya que fue el resultado do la suma del tiempo de actividad uterina sin que hubiera habido modificación cervical.

\section{Permanencia en trabajo en el Instituto}

El único objeto de este aparte es señalar en qué casos se pudo practicar un estudio antes del parto (las que permanecieron todo su trabajo en el servicio), y a quienes se les pudo hacer un estudio retrospectivo, pese a que la gran mayoría permaneció menos de 5 horas en trabajo.

Las agrupamos así:

Permanecieron todo el trabaio de parto 247 casos (36.35).

La permanencia de las demás, se discrimina así:

\begin{tabular}{|c|c|c|}
\hline & Casos & $\%$ \\
\hline Menos de 1 hora ... & 167 & 38.92 \\
\hline De 1 a 5 horas ... & .189 & 44.05 \\
\hline De 1 a 5 horas ... & 189 & 44.05 \\
\hline De 5 a 10 horas ... & 56 & 13.05 \\
\hline Más de 15 horas ... & 2 & 0.46 \\
\hline Sin dato .......... & 2 & 0.46 \\
\hline
\end{tabular}


La menor permanencia fue de $5 \mathrm{mi}$ nutos y la mayor fue de 24.20 horas, paciente que fue a quien se le anotó el mayor tiempo de trabajo.

\section{Tiempo aproximado de muerte fetal}

Sólo se pudo obtener este dato en 290 casos (42.89\%), basados en lo manifestado por la paciente sobre el día y la hora en que dejó de percibir movimientos fetales. Comprobamos que a menor edad del embarazo mayor permanencia del feto muerto in útero y viceversa, apreciación que está de acuerdo con el único informe extranjero que encontramos (10). Además, es notoria la relación con la causa de muerte fetal y así vimos que en los casos de abruptio placentae y toxemia, la retención del feto fue menor.

Los agrupamos así :

\begin{tabular}{|c|c|c|}
\hline & Casos & $\%$ \\
\hline Menos de 48 horas... & 55 & 18.96 \\
\hline De 2 a 5 días ... & 136 & 46.89 \\
\hline De 5 a 8 días ... & 44 & 15.17 \\
\hline De 8 a 12 días $\ldots$. & 17 & 5.86 \\
\hline De 12 a 15 días. & 19 & 6.55 \\
\hline Más de 15 días ... & 19 & 6.55 \\
\hline
\end{tabular}

Primó el dato de 2 a 5 días de permanencia del feto muerto in útero.

El menor tiempo de muerte fetal fue de 6 horas y el mayor, de 60 días.

\section{Conducta}

Son dos las conductas que se han propuesto. Las pasivas, expectantes, o de observación, fundamentadas en el principio de que el feto muerto in útero y la placenta inactiva se comportan como "cuerpos extraños" y que como tales deben incitar al útero a expulsar su contenido, teoría por demás de mucha relatividad $(4,12,13,16)$. Las activas, cuyo principio ha sido el vaciamiento inmediato del útero. Por considerarlo pertinente enumeramos los métodos usuales en la conducta activa y los que recientemente han sido propuestos por la escuela norteamericana (3).

Amniotomía. La consideramos un método de gran utilidad en el refuerzo o conducción del trabajo, apoyados en lo propuesto por la escuela uruguaya (1). Sin embargo, no participamos de ella como método inductivo único, no sólo por considerarla insuficiente, sino porque desconociendo el estado de "modificación” fetal, nos puede hacer agregar un problema al ya instaurado: la infección amniótica consecutiva a la ruptura prematura de membranas. Entre nosotros se usó exclusivamente como elemento conductivo o de refuerzo del trabajo.

Refuerzo del trabajo con ocitócicos. Consideramos que está indicado siempre que haya incoordinación de las contracciones o cualquier otra anomalía de la dinámica por defecto. Es pues un elemento conductivo del trabajo.

Inducción del trabajo con ocitócicos. Debe ser, en principio, la conducta inmediata, verificada la muerte fetal anteparto. Fundamento de lo anterior pueden ser los riesgos maternos que conlleva el feto muerto y retenido entre los cuales vale la pena mencionar los irastornos de la coagulación imputables al paso de líquido amniótico a la circulación 
materna. Además, no hay que olvidar el efecto síquico que acarrea en la mujer el saber que es portadora de un feto muerto.

En cuanto a la técnica inductiva ha de emplearse la infusión continua intravenosa de solución dextrosada que contenga ocitocina, sin predecir cuántas inducciones sea menester aplicar. Por no contar ya el feto podemos usar con más liberalidad la ocitocina, teniendo presente que a menor edad de embarazo mayor cantidad de ocitócicos.

Agrupamos a continuación las conductas entre nosotros:

\begin{tabular}{|c|c|c|}
\hline & Casos & $\%$ \\
\hline Expectantes & 338 & 50 \\
\hline Activas $\ldots \ldots \ldots$. & 338 & 50 \\
\hline
\end{tabular}

Las conductas activas se discriminan así :

\begin{tabular}{lrr}
\hline & Casos & \multicolumn{1}{c}{$\%$} \\
\hline Inducción del trabajo . & 97 & 28.69 \\
Refuerzo del trabajo . & 209 & 61.83 \\
Otras conductas ... . & 32 & 9.46 \\
\hline
\end{tabular}

De los refuerzos de trabajo 159 ( $76.07 \%$ ) fueron sólo amniotomía; 38 (18.18\%) fueron con ocitócicos y 12 $(5.74 \%)$ fueron mixtos.

Las "otras conductas" se refieren a 29 cesáreas, un fórceps, una versión interna y una cesárea con histerectomía inmediata (por C. A. de cuello).

Como se ve en el cuadro, en esta revisión se dividieron equitativamente las conductas, pero cabe anotar que pese a que la morbilidad materna fue mayor en los casos de conducta activa ( 10 de los 14 casos), la gran mayoría de ellos estuvo más relacionada con la causa de muerte fetal que con la conducta misma.

Se practicó una sola inducción en 75 casos; 2 inducciones en 13 casos; 3 inducciones en 5 casos; 4 inducciones en 2 casos; 5 inducciones en 1 caso y 6 inducciones en 1 caso. El resultado de las inducciones fue muy bueno en cuanto a que sólo en el caso en que se practicaron 6 , hubo de hacerse una cesárea por inercia.

En los más recientes informes norteamericanos llegados a nosotros, se propone una conducta de extraordinario interés (3), pues pese a los pocos casos que presenta, bien puede ser una solución al problema de la conducta. Es la inducción del trabajo por inyección intraovularde soluciones isotónicas o hipertónicas, con mucho mejor resultado que la inducción con ocitócicos. Con las soluciones isotónicas se lleva al útero a una sobredistensión que por sí sola inicia el trabajo. Con las soluciones hipertónicas no se alcanza a sobredistender el útero por la menor cantidad usada y serán los cambios de presión intraamniótica, los que desencadenarán el trabajo.

Tipo de parto

Apreciamos el alto porcentaje en que los partos fueron cspontáneos. Esto se explica por el hecho de que la muerte del feto cambia las más de las veces la conducta para el parto y así vemos que en algunas pacientes en que esté indi- 
cada una cesárea, por ejemplo, el hecho de que su feto esté muerto nos hace buscar todos los elementos que ayuden a evitársela. Igual sucede con el fórceps y la ventosa, que todos ellos pierden su indicación fetal y ganan indicaciones maternas, que son menos frecuentes.

La morbilidad materna relacionada con el tipo de parto fue mayor en los partos espontáneos (11 de los 14 casos), tal vez por ser éstos la mavoría, pero en los intervenidos sólo un caso estuvo relacionado con la misma intervención: un desgarro perineal en el caso de un fórceps.

Agrupamos en el siguiente cuadro los tipos de partos, discriminando las intervenciones:

\begin{tabular}{lrrr}
\hline & & \multicolumn{1}{c}{ Casos } & $\%$ \\
\hline Partos espontáneos & $\ldots$ & 590 & 87.27 \\
Partos intervenidos & $\ldots$ & 86 & $\mathbf{1 2 . 7 2}$ \\
\hline
\end{tabular}

Los partos intervenidos se discriminan así :

\begin{tabular}{|c|c|c|}
\hline & Casos & $\%$ \\
\hline Cesáreas ......... & 49 & 56.97 \\
\hline Fórceps $\ldots \ldots \ldots \ldots$ & 20 & 23.25 \\
\hline Embriotomías ...... & 6 & 6.96 \\
\hline Ventosas . . . . . . . & 5 & 5.81 \\
\hline Versión interna ..... & 5 & 5.81 \\
\hline Extracción podálica . . & 1 & 1.16 \\
\hline
\end{tabular}

Causas probables de muerte fetal

En este capítulo, cuyo interés está dado por la complejidad del cuadro, están de acuerdo casi todos los autores $(4,12,13,16)$ al dividirlo en: 1 .
Causas maternas, que pueden ser generales y obstétricas; 2. Causas fetales, y 3. Causas ovulares. Ellas en su mayoría nos recuerdan las mismas del aborto (15).

Por considerarlas todas ellas de interés, haremos una pequeña recapitulación de las que tuvieron participación en este trabajo, resaltando que al igual de los informes extranjeros (10), la primacía está dada por las causas desconocidas.

Causas de muerte fetal:

\begin{tabular}{|c|c|c|}
\hline & Casos & $\%$ \\
\hline 1. Desconocida ... & 237 & 35.05 \\
\hline 2. Toxemia ...... & 164 & 24.26 \\
\hline 3. Accidentes & & \\
\hline del cordón .... & 69 & 10.20 \\
\hline 4. Abruptio & & \\
\hline placentae & 67 & 9.91 \\
\hline 5. Infección & & \\
\hline amniótica $\ldots \ldots$ & 28 & 4.14 \\
\hline 6. Placenta previa. & 27 & 3.99 \\
\hline 7. Postmadurez ... & 18 & 2.66 \\
\hline 8. Lúes ......... & 15 & 2.21 \\
\hline 9. Eritroblastosis .. & 14 & 2.07 \\
\hline 10. Hidramnios ... & 9 & 1.33 \\
\hline 11. Malformaciones & & \\
\hline fetales $\ldots \ldots$ & 7 & 1.03 \\
\hline 12. Diabetes ..... & 5 & 0.73 \\
\hline $\begin{array}{l}\text { 13. Anemia por Ul. } \\
\text { gástr. sangr. . . } \\
\text { 14. Situación }\end{array}$ & 3 & 0.42 \\
\hline transversa $\ldots$. & 3 & 0.42 \\
\hline 15. C. A. de cuello & 2 & 0.28 \\
\hline 16. Gemelaridad ... & 1 & 0.14 \\
\hline $\begin{array}{l}\text { 17. Ruptura espon- } \\
\text { tánea del útero . }\end{array}$ & 1 & 0.14 \\
\hline
\end{tabular}


Encontramos además las siguientes entidades como causa de muerte, cada una de ellas con un caso y que más adelante están consideradas en un solo grupo, junto con la diabetes, el C. A. de cuello y la anemia: T. B. C., neumopatía, angina, bocio tóxico, hipertiroidismo y traumatismo abdominal.

1. Causas desconocidas. Ocupa el primer lugar; su frecuencia fue entre nosotros del $37.05 \%$. Entre los americanos es del $27.8 \%$ (10), quienes anotan que en los 10 años revisados, hay una muerte fetal anteparto cada mes, que ocurre en el último trimestre de embarazo, en pacientes cuyo embarazo había transcurrido normalmente y sin que la causa de muerte fetal pudiera ser explicada. Consideramos que tal afirmación nos debe alertar, puesto que nuestro más alto índice, bien puede deberse a una falta de estudio prenatal (sólo el $8.25 \%$ de las pacientes concurrió a dicha consulta), y más aún, a la falta de estudio en las pacientes a quienes se les ha diagnosticado la muerte fetal. Corrigiendo semejante irregularidad, tal vez se pueda bajar esta incidencia no sólo a la presentada por los americanos sino mucho menos, en consideración a que ellos anotan igualmente falta de elementos diagnósticos, como es la anatomía patológica.

2. Toxemia. La toxemia fue la causa directa de la muerte fetal en el $24.26 \%$ de nuestro grupo, índice menor que el de los americanos $(26 \%)$ (10), pese a que nuestra revisión es mucho más numerosa, lo que nos indica la mejor bondad de nuestro tratamiento en la toxemia. Al igual que ellos, in- cluímos en este grupo todos los tipos de toxemia: preeclampsia, eclampsia, hipertensión y nefritis.

3. Accidentes del cordón. Esta fue la tercera causa probable de muerte fetal anteparto entre nosotros, $10.20 \%$, cuando entre los americanos fue la sexta causa, con 27 casos (10). Estos accidentes son más frecuentes al final del embarazo. Por considerar las causas de crror que ellos pueden determinar, tuvimos en cuenta para incluírlos como causa de muerte fetal, los casos en que el feto nació macerado; aquellos en que se hizo el diagnóstico de procúbito sin que hubiera trabajo y aquellos que por los datos de muerte fetal anteparto dados por la madre, mostraban al cordón, retrospectivamente, como la causa más probable. Discriminamos aquí, por considerarlo de interés, tales accidentes:

Casos

1 Circular .......... 32

2 Circulares ........... 13

3 Circulares ............ 6

Procúbito .......... 5

1 Nudo verdadero ........ 4

Más de 3 circulares ....... 3

Procidencia ........... 2

2 Circulares y 1 nudo ...... 1

1 Circular y 1 nudo ....... 1

2 Nudos verdaderos ........ 1

Torsión ............ 1

En los casos de más de 3 circulares, 2 casos fueron de 4, y 1 fue de 6 .

4. Abruptio placentae. Fue nuestro cuarto gran grupo y forma el 9.91\% entre las causas, a diferencia de los 
americanos, cuyo índice de $14.3 \%$, lo coloca como la tercera causa. En nuestro estudio, el abruptio no respetó la edad del embarazo y estuvo siempre relacionado con la toxemia, menos un caso de traumatismo abdominal, en que bien pudo ocurrir tal accidente (6). En la mayoría de los casos, el feto fue expulsado entre el primero y el segundo día de su muerte.

5. Infección amniótica. Ocupa la amniotitis el quinto puesto en frecuencia, $4.14 \%$, siendo la octava causa entre los americanos (10), que sólo traen 4 casos. Esto es indicio de impreparación en nuestras pacientes, pues todas concurrieron al servicio con el cuadro ya establecido.

6. Placenta previa. Incluímos en este grupo indiscriminadamente, todos los tipos de placenta previa, y fue causa de muerte fetal anteparto en el $3.99 \%$ de los casos. Los americanos no traen esta causa en el grupo que estudiaron, y por el hecho de ser esta entidad más frecuente que otras traídas por ellos, bien pudo tratarse de una omisión (8).

7. Postmadurez. La muerte anteparto del feto después de la semana 42 (17), la encontramos en el $2.66 \%$ de los casos, y los americanos (10) presentan sólo 3 casos en su estudio. Pese a que la frecuencia de embarazos de más de 40 semanas fue superior, la mayoría de ellos están incluídos en otros grupos por estar asociadas a causas más probables de la muerte fetal.

8. Lúes. Relacionamos la muerte fetal anteparto con la sífilis en el $2.21 \%$ de los casos. En el trabajo de los ameri- canos (10) no aparece esta causa, a diferencia, como ellos anotan, de la gran incidencia encontrada en trabajos de décadas anteriores.

9. Eritroblastosis. Se relacionó esta entidad con la muerte del feto en el $2.07 \%$ de los casos. Los americanos (10) la tienen en quinto grupo por cu frecuencia $(8.8 \%)$. No hicimos distinción entre incompatibilidad por $\mathrm{Rh}$ y por grupo. Sólo encontramos una paciente de este grupo con antecedentes de un feto muerto.

10. Polihidramnios. En el $1.33 \%$ de los casos relacionamos esta entidad con la muerte del feto. Los americanos (10) la incluyen en el grupo de anomalías fetales, que es el cuarto grupo de ellos en frecuencia y presentan 12 casos, índice bastante superior al nuestro.

1. Malformaciones fetales. Agrupamos aquí todos aquellos casos en que la única causa relacionada con la muerte del feto fueron sus anomalías congénitas. Su frecuencia fue del $1.03 \%$, menor que la de los americanos (10), que fue del $11.1 \%$, así se deduzcan los casos de polihidramnios por ellos incluídos en este capítulo. Discriminamos las malformaciones como sigue:

Casos

Múltiples anomalías ... . . . $\quad 2$

Hidrocefalia $\ldots \ldots \ldots \ldots \ldots \quad 2$

Anencefalia $\ldots \ldots \ldots \ldots \ldots 1$

Microcefalia ......... 1

Anasarca $\ldots \ldots \ldots \ldots \ldots \quad 1$

Suponemos que la falta de estudio anatomopatológico haya hecho pasar 
desapercibidas otras tantas anomalías, como pudieran ser cardiopatías, malposiciones orgánicas, etc.

12. Situación transversa. Se presentó como causa probable de muerte fetal en 3 casos $(0.42 \%)$. Por la duda que puede despertar tal eventualidad como causa de muerte fetal, aclaramos que los 3 casos se consideran como tales por tratarse uno de un primer gemelo en situación transversa y los otros 2 por haber sido fetos macerados, ambos extraídos por versión interna.

13. Ruptura espontánea del útero. Hubo 1 caso en que pudimos relacionar tal accidente con la muerte del feto, pues sucedió en una paciente con cesárea previa que ingresó al servicio con feto muerto, sin trabajo. Por haber presentado en las 24 horas siguientes un cuadro de distensión abdominal por pseudooclusión intestinal, se instauró tratamiento para ello; al no ceder dicho cuadro al tratamiento médico, al $4^{\circ}$ día del ingreso se resolvió practicar laparotomía y cesárea. Se encontró dehiscencia de la cicatriz de la histerotomía y una peritonitis. Esta paciente murió en el postoperatorio.

14. Gemelaridad. Encontramos 1 caso en donde la única causa probable de muerte fetal fue la gemeralidad; en este caso murió el segundo gemelo y nació macerado.

15. Enfermedades generales maternas. Incluímos en este grupo todas las entidades médicas que vimos estrechamente relacionadas con la muerte del feto. Se atribuyó así la muerte del feto a: 5 casos de diabetes $(0.73 \%), 3$ ca- sos de anemia por úlcera gástrica sangrante $(0.42 \%), 2$ casos de C. A. de cuello $(0.28 \%)$ y sendos casos de tuberculosis, neumopatía, angina, bocio tóxico, hipertiroidismo y traumatismo abdominal.

Aquí llama la atención el escaso número de diabetes y tal vez el grande de úlceras gástricas.

Podemos recalcar que en el trabajo americano (10) se agrupan en 10 las causas de muerte fetal, justificando así la complejidad del tema. Nosotros lo hicimos en 15 grupos, contando en el último de ellos varias entidades relacionadas todas con la muerte del feto. Insistimos que un estudio más cuidadoso nos autorizará a hablar de "Causas de muerte fetal" y no de "Causas probables de muerte fetal", como hasta ahora hemos venido haciendo.

Peso fetal

Consideramos de valor este dato, pues como se ve en el cuadro siguiente, la mavor frecuencia de la muerte fetal anteparto está entre los 1.500 y los 2.500 grs. Por otra parte se recordará que la mayor edad gestacional fue 3640 semanas y el tiempo de retención máximo de 2 a 5 días; es decir, algo más o menos contradictorio.

\begin{tabular}{lrrr}
\hline & Casos & \multicolumn{1}{c}{$\%$} \\
\hline De 1.500 a 2.000 grs. & 204 & 30.08 \\
De 2.001 a 2.500 ”, & 208 & 30.67 \\
De 2.501 a 3.000 ” & 146 & 21.53 \\
De 3.001 a 3.500 ” & 62 & 0.14 \\
De 3.501 a $4.000 ”$ & 15 & 2.21 \\
Más de 4.000 .... & 10 & 1.47 \\
Sin dato ........ & 33 & 4.86 \\
\hline
\end{tabular}


El feto de mayor peso fue de 5.400 gramos.

\section{Morbilidad materna}

Fue del $2.07 \%$. Debemos resaltar que en ningún caso pudimos relacionar la complicación morbosa con el feto muerto.

Casos

Fibrinogenopenia $\ldots \ldots \ldots 5$

Puerperio febril ......... 3

Desgarro perineal ......... 2

Hemorragia del alumbramiento . 2

Tromboflebitis .......... 1

Insuficiencia renal $\ldots \ldots \ldots 1$

Los 5 casos de fibrinogenopenia estuvieron relacionados, 3 de ellos con abruptio placentae y los otros 2 con toxemia (eclampsia). De los 3 puerperios febriles, 2 tuvieron que ver con infección amniótica. La insuficiencia renal y una de las hemorragias del alumbramiento, con el abruptio.

\section{Estado fetal al nacer}

Consideramos en este capítulo la maceración fetal, sin tener en cuenta sus grados por no estar consignados en las historias. Tuvimos en la maceración el signo retrospectivo más evidente de muerte fetal anteparto, cuando hubo dudas al respecto.

\begin{tabular}{ccc}
\hline & Casos & $\%$ \\
\hline Se presentó maceración & & \\
en . . . . . . . . . & 492 & 72.56 \\
No hubo evidencia en & 186 & 27.43 \\
\hline
\end{tabular}

Sólo hubo un feto en estado de putrefacción, que está incluído entre los macerados, fruto de una infección amniótica con C. A. de cuello.

\section{Mortalidad materna}

Consideramos ésta pese a que en ningún caso pudimos relacionarla estrictamente con el estado de muerte fetal anteparto. Su índice fue del $1.33 \%$ y se discrimina así:

Casos

Hemorragia cerebral ....... 2

C. A. de cuello .......... 1

Sepsis por amniotitis ....... 1

Peritonitis por ruptura uterina . . 1

Anemia aguda por placenta cen-

tral ............ 1

Glomerulonefritis ......... 1

Insuficiencia renal ........ 1

Bronconeumonía lobar bilateral . 1

Apenas quedaría la duda de la amniotitis en cuanto a si hubo conducta errónea o tardía. Se practicó necropsia en solo cuatro casos.

\section{RESUMEN}

Se hace una revisión de la muerte fetal anteparto en el Instituto Materno Infantil "Concepción Villaveces de Acosta" del 10 de marzo de 1959 al 28 de febrero de 1963 entre fetos de más de 1.500 gramos.

De 50.420 partos, 678 fetos murieron antes del parto $(1.34 \%)$ en 676 pacientes.

Las causas probables más importantes de la muerte fetal fueron: de causa 
desconocida ( $35.05 \%)$, t o x e m i a $(24.26 \%)$, accidentes del c or dón $(10.20 \%)$, abruptio placentae (9.91 por ciento), infección amniótica (4.14 por ciento), placenta previa (3.99\%), postmadurez $(2.66 \%)$, eritroblastosis $(2.07 \%)$.

El diagnóstico de la muerte se basó en la auscultación del corazón fetal, en el signo de Negri, en la ausencia de movimientos fetales, en la calostrorrea y en los signos obtenidos mediante el empleo de rayos $\mathrm{X}$.

En el $50 \%$ de los casos la conducta fue expectante y en el resto activa (inducción, cesáreas).

En el $87.27 \%$ el parto se hizo en forma espontánea y en el $12.72 \%$ fue intervenido (cesáreas, fórceps, embriotomías, ventosas, etc.).

La morbilidad materna fue del 2.07 por ciento ( 14 casos) y se comprueba que ella en su totalidad no tuvo relación con la muerte del feto.

La mortalidad fue del $1.33 \%$, y salvo una tal vez dudosa, en ningún caso estuvo relacionada con la muerte del feto.

\section{CONCLUSIONES}

1. La muerte fetal sigue siendo un problema cuya complejidad no sólo está demostrada en la gran incidencia de causas desconocidas, sino en la falta de estudio de las pacientes.

2. El estudio de estas pacientes se debe enfocar hacia la investigación de la causa de muerte fetal, hacia el diagnóstico de muerte fetal y hacia la con- ducta que ha de seguirse una vez comprobado el diagnóstico.

3. Es mayor la incidencia de muerte fetal entre los 21 y los 25 años y en pacientes multíparas.

4. El feto muere antes del parto con más frecuencia después de las 36 semanas de gestación; en cambio ìos pesos más frecuentes fueron de 1.500 a 2.500 gramos.

5. Se deben ampliar los elementos de juicio, con miras al diagnóstico de muerte fetal y su etiología.

6. El tiempo de retención del feto muerto in útero está en razón inversa a la edad del embarazo.

7. En la toxemia y el abruptio placentae la retención del feto tiende a ser menor que en otras entidades causales.

8. La conducta en el feto muerto debe tender a la vaciamiento inmediato del útero, para lo cual aconsejamos la inducción con ocitócicos una vez hecho el diagnóstico, ya que no tenemos experiencia en la inducción con otros métodos.

9. El número de inducciones necesarias y la cantidad de ocitocina está en razón inversa a la edad del embarazo y al tiempo de retención.

10. Existe mayor tendencia entre nosotros a dejar evolucionar el parto espontáneamente y no hay observación alguna de haber recurrido a tratamiento quirúrgico obstétrico por distocia de cuello o alguna otra causa.

11. Entre nosotros las causas desconocidas, la toxemia, los accidentes del cordón y el abruptio placentae son los 
factores más frecuentes de muerte fetal anteparto.

12. La diabetes no tuvo la incidencia de algunas estadísticas, tal vez por falta de diagnóstico preciso. En cambio fue notoria para sífilis, hipermadurez, eritroblastosis, hidramnios y hasta úlcera gástrica sangrante.

13. La muerte del feto anteparto no se relacionó directamente en ningún caso, con la morbimortalidad materna.

\section{BIBLIOGRAFIA}

1. ALVAREZ CALDEYRO-BARCIA: Fisiopatología de la contracción uterina y sus aplicaciones en la Clínica Obstétrica. Fac. Med. Montevideo, Uruguay.

2. BORELL ULF y col.: The Halo Sign in the living and dead fetus. Am. J. Obst. and Ginec., vol. 87: 7-1963, pág. 906.

3. CSAPO A. I. y col.: Fetal death in utero. Am. J. Obst. and Ginec. vol. 87: 7, 1963, pág. 892.

4. DEXEUS FONT S.: Tratado de Obstetricia, segunda edición. Salvat 1957, págs. 240 a 243 y 1143 a 1151.

5. Departamento de Estadística: Instituto Materno Infantil "Concepción Villaveces de Acosta", Códigos O. M. S. 7×2-001 y 7×2-005.

6. DYER ISADORE, BARCLAY DAVID L.: Accidental Trauma complicating pregnancy and delivery. Am. J. Obst. and Ginec., vol. 83: 7, pág. 907, 1962.

7. Encyclopedie Medico-Chirurgicale, Obstetrique: Souffrance et Mort du Foetus "in utero", premiere éd., 5-1: 958 C, 5077 A, pág. 1 y 5077 A, 30, pág. 1.

8. FOOTE WILLIAM R., FRASER WILLIAM D.: Placenta previa. Am. J. Obst. and Ginec, vol. 80: 1, 1960, pág. 10.

9. FRIEDMAN ROBERT L., MEYER PAUL D.: Intrafetal Gas in utero. Am. J. Obst. and Ginec., vol. 82: 6, 1961, pág. 1397.

10. GRANDUN DEAN J., HALL ROBERT E.: Fetal death, before the onset of labor. Am. J. Obst. and Ginec. vol. 79: 2, pág. 237, 1960.

11. HON E. H., HESS O. W.: The clinical value of fetal electrocardiograhpy. Am. J. Obst. and Ginec., vol. 79: 5, pág. 1021, 1960.

12. LULL CLIFFORD B.: Obstetricia Clínica, Edit. Interamericana S. A., México 1954, pág. 366.

13. MORAgues BERNAT J.: Clínica Obstétrica, 2a edic. Editorial Ateneo, Buenos Aires, 1945, págs. 372 a 377.

14. PERALTA CAYON RAFAEL y col.: Electrocardiograma fetal intrauterino. Rev. Col. Obst. y Gin., vol. IX: 4, pág. 371 .

15. PERALTA CAYON RAFAEL: Aborto-cesárea previas. Controversias Clinicas y Terapéuticas, 1962, pág. 13.

16. PEREZ MANUEL LUIS: Compendio de Obstetricia. Edit. López y Etchegoyen, SRL., Buenos Aires, 1956, págs. 388 a 391.

17. PERLIN I. A.: Postmaturity. Am. J. Obst. and Ginec., vol. 80: 1, 1960, pág. 1.

18. SCHMIDT O. A. y col.: An evaluation of fetal electrocardiography. Am. J. Ohst. and Ginec., vol. 83: 4, 1962, pág. 464. 